\title{
FIELD QUALITY OF LHC/SACLAY ARC QUADRUPOLE MAGNET PROTOTYPE
}

\author{
A. Devred, J. Belorgey, J. Deregel, B. Gallet, P. Genevey, J.M. Rifflet, J.Cl. Sellier, P. Védrine \\ CEA Saclay, DSM/DAPNIA/Service Technique de Cryogénie et de Magnétisme \\ F-91191 Gif-sur-Yvette Cedex, France
}

J. Billan

CERN, LHC Division, 1211 Geneva 23, Switzerland

\begin{abstract}
As part of the magnet R\&D program for the Large Hadron Collider (LHC), CEA Saclay has designed and built two 3-m-long, 56-mm-twin-aperture arc quadrupole magnet prototypes. Extensive harmonic field measurements were performed during the cold test of the second prototype, using a newly developed magnetic measurement system. After a brief description of the new system, a review of the field quality data as a function of axial position and current is presented.
\end{abstract}

\section{INTRODUCTION}

The LHC will require about 400 superconducting twin-aperture quadrupole magnets to focus the counterrotating proton beams. A collaboration program was set up between CEA Saclay and CERN at the end of 1989 to develop two full-length quadrupole magnet cold masses according to the so-called pink book parameters $[1,2]$. The two 3-m-long, 56-mm-twin-aperture cold masses were completed mid-1993 [3,4] and were cold tested in a helium superfluid bath at the Saclay horizontal test facility [5]. They both reached the pink-book nominal current of $15060 \mathrm{~A}$ (corresponding to a $10 \mathrm{~T}$ dipole field and a $252 \mathrm{~T} / \mathrm{m}$ quadrupole gradient) after a limited number of training quenches [6,7]. The first cold mass was then shipped to CERN to be cryostated for a string test along with two dipole magnets [8], while the second cold mass was kept at Saclay to undergo magnetic measurements. After a series of unsuccessful attempts, it was decided in January 1995 to develop a new magnetic measurement equipment. The new system was operational in June 1995. We report here on the field quality data taken during the final cold test of the second LHC/Saclay quadrupole magnet prototype.

The LHC/Saclay quadrupole magnet design relies on $\cos (2 \theta)$ conductor distributions. The coil assemblies are mechanically constrained by laminated collars made of supposedly non-magnetic stainless steel (316 LN). There are two identical collared-coil assemblies per cold mass surrounded by a laminated, low carbon steel yoke. The cold mass is completed by a stainless steel inertia tuble which also serves as helium containment vessel. The collared-coil assemblies use separate collars, while the yoke laminations are common and single-piece. Most of the assembly processes are carried out vertically.

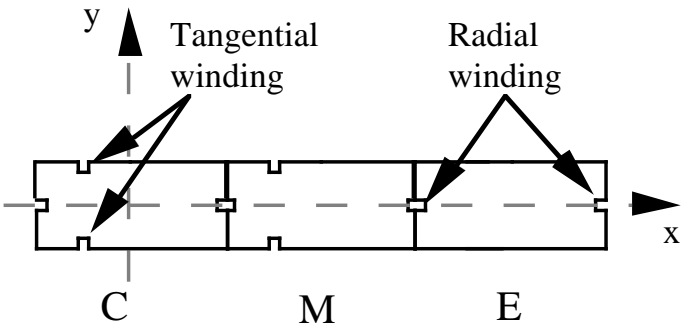

Fig. 1 Schematic view of the rotating coil array used in the Saclay magnetic measurement system.

\section{MULTipole EXPANSION}

In the quadrupole magnet straight section, the field can be considered as two dimensional and is conveniently represented by a multipole expansion

$$
B_{\mathrm{y}}+\mathrm{i} B_{\mathrm{x}}=\sum_{n=1}^{+\infty}\left(B_{\mathrm{n}}+\mathrm{i} A_{\mathrm{n}}\right)\left(\frac{x+\mathrm{i} y}{R_{\text {ref }}}\right)^{\mathrm{n}-1},
$$

where $B_{\mathrm{X}}$ and $B_{\mathrm{y}}$ are the $x$ - and $y$-components of the field, $B_{\mathrm{n}}$ and $A_{\mathrm{n}}$ are the normal and skew $2 n$-pole field coefficients and $R_{\text {ref }}$ is the reference radius. (For LHC magnets, $\left.R_{\text {ref }}=1 \mathrm{~cm}\right)$. The symmetries of a $\cos (2 \theta)$ conductor distribution are such that only the $B_{4 \mathrm{k}+2}$ field coefficients, also called allowed multipole field coefficients, are expected to be non zero.

\section{Magnetic Measurement System}

The new magnetic measurement system developed by CEA Saclay consists of a rotating array of 3 radial coils, referred to as external (E), medium $(\mathrm{M})$ and central $(\mathrm{C})$ and of 2 tengential coils, referred to as $T_{\mathrm{M}}$ and $T_{\mathrm{C}}$ (see Fig. 1). The 50-turn radial coils were wound at CERN on identical, precisely machined mandrels made of epoxyimpregnated fiberglass, using a $0.1 \times 0.8-\mathrm{mm}$ flat cable made of 10 parallel wires connected in series at the coil ends [9]. The 2-turn tangential coils were added at Saclay using a $0.1-\mathrm{mm}$ round wire. The mandrels are about $700 \mathrm{~mm}$ in length. The rotating array is driven externally by a step-by-step motor coupled angularly to a set of 1m-long, epoxy-impregnated fiberglass shafts. The rotation is supported by ceramic bearings. An angle encoder is mounted at the coil array end opposite to the driving shaft. The rotation period is $5 \mathrm{~s}$. 
The CAMAC-based data acquisition system is controlled by a PC. The 5 coils are connected to a voltage switching unit which is configurated by software. It allows the read-out of a number of coil combinations and includes several stages of programmable gain amplifiers. The output signal is sent to a voltage-to-frequency convertor which integrates the signal between trigger pulses given by the angle encoder. Data recorded over a $360^{\circ}$ rotation are processed by mean of a discrete Fourier transform and converted into multipole coefficients.

The parameters of the 3 radial coils are chosen to buck-out the dipole and quadrupole terms from (E$2 \mathrm{M}+\mathrm{C})$. After adjustment of the amplifiers gains, the dipole bucking ratio, $f_{1}$, defined as the ratio of the dipole term in the bucked signal to that in $\mathrm{E}$ alone, is 850 while the quadrupole bucking ratio, $f_{2}$, defined similarly for the quadrupole terms, is 400 . Adding the tangential coils $T_{M}$ and $\mathrm{T}_{\mathrm{C}}$, which are meant to correct for radial coil misalignment, allows to bring $f_{1}$ in excess of 5000 and $f_{2}$ in excess of 25000. Bucking is needed to reduce the effects of mechanical perturbations on the measurements. The reproducibility on $A_{3}$ and $B_{3}$ is a few $10^{-6} B_{2}$.

\section{Geometric Multipoles}

As we have said, only the $B_{4 \mathrm{k}+2}$ field coefficients are expected to be non-zero. In practice, however, manufacturing errors result in conductor positioning which violates the $\cos (2 \theta)$ symmetries and leads to non-zero unallowed multipole field coefficients. The geometric field errors can be estimated by performing measurements at a constant current. The current value must be: 1) high enough so that the contributions from the persistent magnetization currents in the superconductor are negligible and 2) low enough so that the effects from iron yoke saturation are also negligible. The data discussed below were taken at $10 \mathrm{kA}$ (following an up-ramp).

Table 1 presents a summary of the integrated multipole coefficients over the length of the second LHC/Saclay quadrupole magnet prototype. The magnet coordinate system (MCS) is defined by facing the magnet from the non-connection end. (The non-connection end is the magnet end opposite to that where current leads feed the magnet coils.) The $x$-axis is horizontal, oriented from left to right, the $y$-axis is vertical, oriented from bottom to top. The North aperture is on the left-hand side and the South aperture is on the right-hand side. The magnetic measurement system was introduced from the nonconnection end. For each aperture, the measurements were taken at five adjacent positions along the magnet length. The start angle of the rotating coil array was always around zero in the MCS. For each position, the data are reported in a coordinate system, whose $x$-and $y$-axes are approximately parallel and of same direction as that of the MCS, and where the normal and skew dipole field coefficients and the skew quadrupole field coefficient are forced to be zero. This centers and aligns the measurements with respect to the main quadrupole field.
Table 1. Integrated multipole coefficients over the length of the 2nd LHC/Saclay 3-m-long, 56-mm-twin-aperture quadrupole magnet prototype (at $10 \mathrm{kA}$ and $1 \mathrm{~cm}$ ).

\begin{tabular}{c|c|c||c|c|c} 
& North & South & & North & South \\
\hline \hline$a_{3}$ & +0.39 & +0.45 & $b_{3}$ & +0.25 & -0.33 \\
$a_{4}$ & -0.33 & -0.10 & $b_{4}$ & +0.08 & +0.03 \\
$a_{5}$ & -0.05 & +0.05 & $b_{5}$ & -0.01 & -0.06 \\
$a_{6}$ & -0.04 & -0.00 & $b_{6}$ & -0.24 & -0.22 \\
$a_{7}$ & +0.00 & +0.00 & $b_{7}$ & +0.01 & +0.00 \\
$a_{8}$ & -0.00 & -0.00 & $b_{8}$ & +0.00 & -0.00 \\
$a_{9}$ & -0.00 & +0.00 & $b_{9}$ & -0.00 & -0.00 \\
$a_{10}$ & -0.00 & -0.00 & $b_{10}$ & -0.01 & -0.01
\end{tabular}

The integrated coefficients, $a_{\mathrm{n}}$, are estimated as

$$
a_{\mathrm{n}}=10^{4}\left(\sum_{i=1}^{5} A_{\mathrm{n}}^{\mathrm{i}} L_{\mathrm{i}}\right) /\left(\sum_{i=1}^{5} B_{2}^{\mathrm{i}} L_{\mathrm{i}}\right)
$$

where $A_{\mathrm{n}}^{\mathrm{i}}$ and $B_{\mathrm{n}}^{\mathrm{i}}$ are the center and phase-corrected $2 n$ pole field coefficients at position $i$, in average over a counter-clockwise and a clockwise rotation, and $L_{\mathrm{i}}$ is the effective measuring coil length at position $i$. Similar definitions are used for $b_{\mathrm{n}}$.

The integrated values presented in Table 1 are quite small, especially for high order multipole coefficients. Looking at the details of the measurements as a function of position, there appears to be little variations over the magnet length, except for the normal sextupole coefficient in the South aperture, which exhibits peak to peak variations of the order of $1.5 \times 10^{-4} B_{2}$, and for the allowed multipole coefficients (normal dodecapole and normal 20-pole) which present end effects. The coil ends have been optimized, but their contributions were not set rigorously at zero.

\section{Measurements as a FunCtion OF CURRENT}

Persistent magnetization currents are generated at the periphery of the superconducting filaments each time the current is ramped to shield the filaments' cores from local field variations. The filaments, with their shells of magnetization currents, then behave as magnetic doublets which distort the central field. However, since the doublets' orientation is determined by the transport-current field, only the allowed multipole coefficients are affected. These effects are only sensible at low fields. At high fields, the iron yoke starts to saturate, but, in the case of the LHC/Saclay quadrupole magnet design, the computed distorsions of the multipole coefficients are negligible.

Figures 2(a) and 2(b) presents summary plots of $b_{6}$ and $b_{10}$ as a function of current. The measurements were taken at the axial center (position 3) of each aperture during the successive plateaus of a staircaise-type current loop. This loop was preceeded by a standardization cycle to $13 \mathrm{kA}$. The system set-up and the data processing are the same as for the measurements discussed previously. 

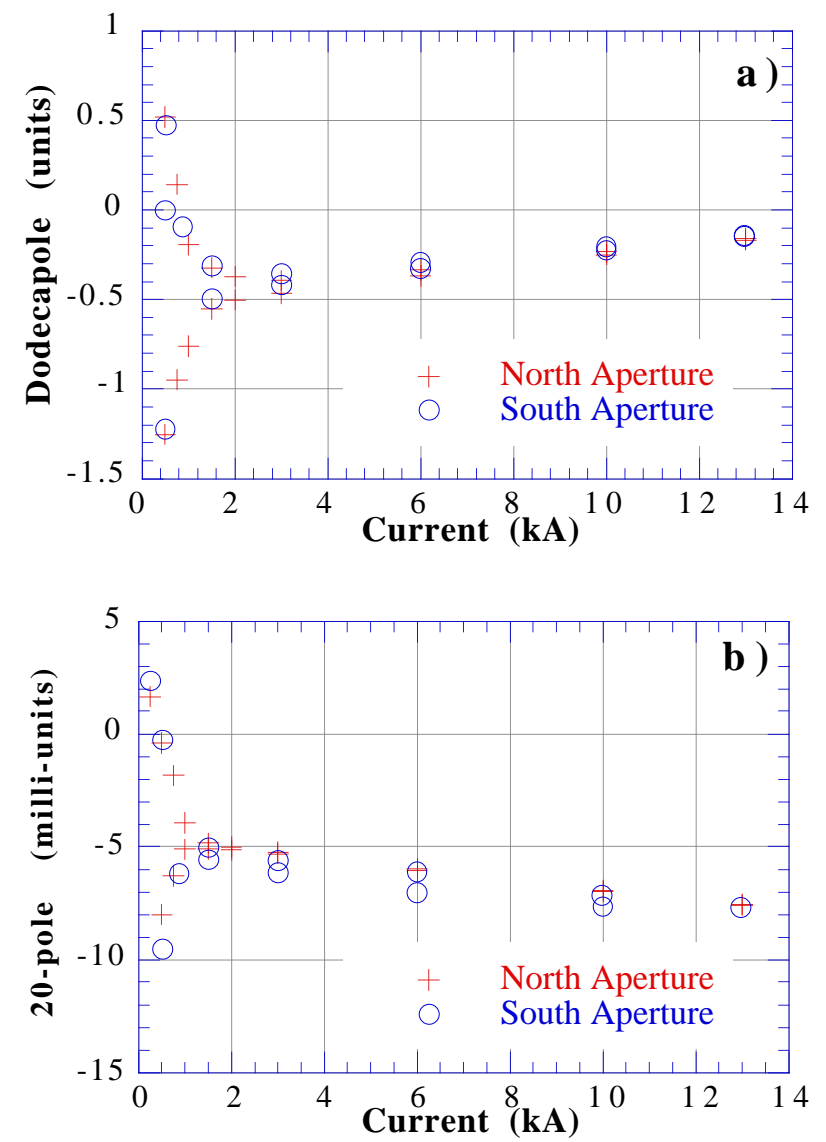

Fig. 2. Dimensionless multipole coefficients versus current: a) dodecapole $\left(b_{6}\right)$ and b) 20-pole $\left(b_{10}\right)$.

The dimensionless multipole coefficients, $b_{\mathrm{n}}$, are defined as

$$
b_{\mathrm{n}}=10^{4} B_{\mathrm{n}} / B_{2},
$$

where $B_{\mathrm{n}}$ is the center and phase-corrected normal $2 n$-pole field coefficients at the given position and current, in average over a counter-clockwise and clockwise rotation.

The data plotted on Fig. 2 present three remarkable features: 1) $b_{6}$ and $b_{10}$ exhibit hysteretic behaviors, 2) the average values of the hystereses vary linearly and 3) the behaviors are identical for the two apertures.

The observed hystereses are consistent with what can be expected from the effects of persistent magnetization currents. The half-width of the $b_{6}$ hysteresis (estimated from up-ramp minus down-ramp data) is around -0.35 units for a current of $850 \mathrm{~A}$ (which corresponds to a normal quadrupole field at $1 \mathrm{~cm}$ of $0.143 \mathrm{~T}$ ), while that of the $b_{10}$ hysteresis is less than +0.01 units. The pink book injection current of the machine was set to $875 \mathrm{~A}$.

The variations of the average values of the hystereses were not expected. A likely explanation for this behavior is the fact that the stainless steel collars are slightly magnetic. The collar magnetic permeability was specified to be less than 1.02, but was measured to decrease from 1.02 to 1.01 when the field is increased from 1 to $5 \mathrm{~T}$. Finite element computations show that such a field dependence of the collar permeability results in a quasi- linear $b_{6}$ increase of 0.25 units and a quasi-linear $b_{10}$ decrease of 0.002 units when the current is increased from 3 to $13 \mathrm{kA}$. These computated variations are in reasonable agreement with the observed ones.

In addition, a test was performed to see the effects of a current imbalance between the two apertures. For this test, a secondary power supply was implemented in parallel to the South aperture. Let $I_{\mathrm{m}}$ designate the current delivered by the main power supply. The North aperture current was always $I_{\mathrm{m}}$ while that in the South aperture was increased up to $I_{\mathrm{m}}+3 \mathrm{kA}$. As expected from computations, a current imbalance of up to $3 \mathrm{kA}$ appears to have no measurable effects on the North aperture multipole coefficients. In the new LHC lattice, it is envisioned to connect the quadrupoles apertures on two separate buses.

\section{CONCLUSION}

Except for the magnetic properties of the stainless steel collars, the field quality of the second LHC/Saclay quadrupole magnet prototype is acceptable. Work has now begun on the development of two additional prototypes according to the new LHC lattice parameters.

\section{ACKNOWLEDGMENT}

The authors are greatly indebted to J. Pérot for his contribution to the developement of particle accelerator magnets and for his continuous support of this work.

\section{REFERENCES}

[1] The LHC Study Group, "Design study of the Large Hadron Collider (LHC)", CERN 91-03 (1991).

[2] J.M. Baze, D. Cacaut, et al., "Design and fabrication of the prototype superconducting quadrupole for the CERN LHC Project", IEEE Trans. Magn., Vol. 28(1), pp. 335337 (1992).

[3] J.M. Rifflet, P. Giovannoni, et al., "Status of the fabrication and tests of the prototype LHC lattice quadrupole magnets", IEEE Trans. Magn., Vol. 30(4), pp. 2458-2461 (1994).

[4] P. Vedrine, J.M. Rifflet, et al., "Mechanical tests on the prototype LHC lattice quadrupole", IEEE Trans. Magn., Vol. 30(4), pp. 2475-2478 (1994).

[5] P. Chaumette, C. Curé, et al., "A large $1.8 \mathrm{~K}$ facility for magnet tests", Adv. Cryo. Eng., Vol. 35(A), pp. 269274 (1990).

[6] J.M. Rifflet, J. Cortella, et al., "Cryogenics and mechanical measurements of the first two LHC lattice quadrupole prototypes," Proceedings of the 4th European Particle Accelerator Conference, World Scientific, pp. 2265-2267 (1994).

[7] P. Genevey, J. Deregel, et al., "Cryogenic tests of the first two LHC quadrupole prototypes, IEEE Trans. Appl. Super., Vol. 5(2), pp. 202-205 (1995).

[8] F. Rodiguez-Mateos, L. Coull, et al., "Electrical performance of a string of magnets representing a halfcell of the LHC machine," presented at the 14th International Conference on Magnet Technology, Tampere, Finland, June 11-16, 1995.

[9] S. Bidon, J. Billan, et al., "New technique of fabrication of search coil for magnetic field measurement by harmonic analysis," Proceedings of the 9th International Magnet Measurement Workshop, CEA Saclay, Orme des Merisiers, June 19-22, 1995. 